\title{
Spatial distribution and spectral characteristics of energetic electrons in Mercury's magnetosphere
}

\author{
George C. Ho, ${ }^{1}$ Stamatios M. Krimigis, ${ }^{1,2}$ Robert E. Gold, ${ }^{1}$ Daniel N. Baker, ${ }^{3}$ \\ Brian J. Anderson, ${ }^{1}$ Haje Korth, ${ }^{1}$ James A. Slavin, ${ }^{4}$ Ralph L. McNutt Jr., ${ }^{1}$ \\ Reka M. Winslow, ${ }^{5}$ and Sean C. Solomon ${ }^{6,7}$ \\ Received 31 May 2012; revised 11 July 2012; accepted 20 July 2012; published 7 September 2012.
}

[1] The Energetic Particle Spectrometer (EPS) on the MESSENGER spacecraft, in orbit about Mercury since March 2011, has detected bursts of low- and moderate-energy (tens to hundreds of $\mathrm{keV}$ ) electrons during portions of most orbits. There have been periods when such bursts were observed regularly on every orbit over a span of several weeks, and other periods when electrons were not observed for several days at a time. We have systematically characterized these energetic events on the basis of particle intensity over the 12-month period since MESSENGER began orbital operations. Now that MESSENGER has sampled most Mercury longitudes and local times, it is evident that the largest burst events were either at high northern latitudes or near local midnight. Lower-energy events were also seen near the equator but were mostly absent in both the dawn and dusk local time sectors. The high-latitude and nightside events are similar in particle intensity, spectra, and pitch angle and are interpreted to be the result of acceleration by the same mechanism. Another group of events occurred upstream of Mercury's bow shock. For two examples of this group of upstream events with good pitch angle coverage, the particles were field-aligned and traveling away from the bow shock. This group of events is interpreted to be similar to upstream events found at Earth during which particles are accelerated at the bow shock and subsequently travel upstream into the solar wind.

Citation: Ho, G. C., S. M. Krimigis, R. E. Gold, D. N. Baker, B. J. Anderson, H. Korth, J. A. Slavin, R. L. McNutt Jr., R. M. Winslow, and S. C. Solomon (2012), Spatial distribution and spectral characteristics of energetic electrons in Mercury's magnetosphere, J. Geophys. Res., 117, A00M04, doi:10.1029/2012JA017983.

\section{Introduction}

[2] On 18 March 2011 the MErcury Surface, Space ENvironment, GEochemistry, and Ranging (MESSENGER) spacecraft entered orbit about Mercury and began the primary phase of its mission. Prior to orbit insertion the spacecraft completed three flybys of the innermost planet in 2008 and 2009 [McNutt et al., 2010; Solomon, 2011]. There

\footnotetext{
${ }^{1}$ Johns Hopkins University Applied Physics Laboratory, Laurel, Maryland, USA.

${ }^{2}$ Office of Space Research and Technology, Academy of Athens, Athens, Greece.

${ }^{3}$ Laboratory for Atmospheric and Space Physics, University of Colorado Boulder, Boulder, Colorado, USA.

${ }^{4}$ Department of Atmospheric, Oceanic and Space Sciences, University of Michigan, Ann Arbor, Michigan, USA.

${ }^{5}$ Department of Earth and Ocean Sciences, University of British Columbia, Vancouver, British Columbia, Canada.

${ }^{6}$ Department of Terrestrial Magnetism, Carnegie Institution of Washington, Washington, D. C., USA.

${ }^{7}$ Lamont-Doherty Earth Observatory, Columbia University, Palisades, New York, USA.

Corresponding author: G. C. Ho, Johns Hopkins University Applied Physics Laboratory, Laurel, MD 20723, USA. (george.ho@jhuapl.edu)

(C)2012. American Geophysical Union. All Rights Reserved. 0148-0227/12/2012JA017983
}

were no energetic (energy $E>35 \mathrm{keV}$ ) particles detected by the MESSENGER Energetic Particle Spectrometer (EPS) during any of the three flybys [Ho et al., 2011a]. One of two sensors within the Energetic Particle and Plasma Spectrometer (EPPS) instrument, EPS measures electrons with energies from $\sim 35 \mathrm{keV}$ to $\sim 1 \mathrm{MeV}$ [Andrews et al., 2007]. The absence of energetic particle bursts during the MESSENGER flybys was in contrast to a report from the first Mariner 10 flyby of Mercury in 1974 that impulsive high fluxes of both energetic electrons $(>175 \mathrm{keV})$ and ions $(>550 \mathrm{keV})$ were detected inside Mercury's small magnetosphere [Simpson et al., 1974]. However, from fluorescent X-ray events seen by MESSENGER's X-Ray Spectrometer (XRS) during the three flybys, Ho et al. [2011a] showed that suprathermal plasma (tens of $\mathrm{keV}$ ) electrons were present inside Mercury's magnetosphere at energies below the EPS energy threshold. Armstrong et al. [1975, 1979], Christon [1989], and Ho et al. [2011a] suggested that the Mariner 10 energetic particle instruments were likely responding to pulse pile-up from lower-energy $(\sim 35-175 \mathrm{keV})$ electrons rather than more energetic $(>175 \mathrm{keV})$ electrons or ions.

[3] Shortly after MESSENGER entered orbit about Mercury and instruments were turned on, EPS detected recurrent bursts of energetic electrons within Mercury's magnetosphere [Ho et al., 2011b]. These bursts were of short 


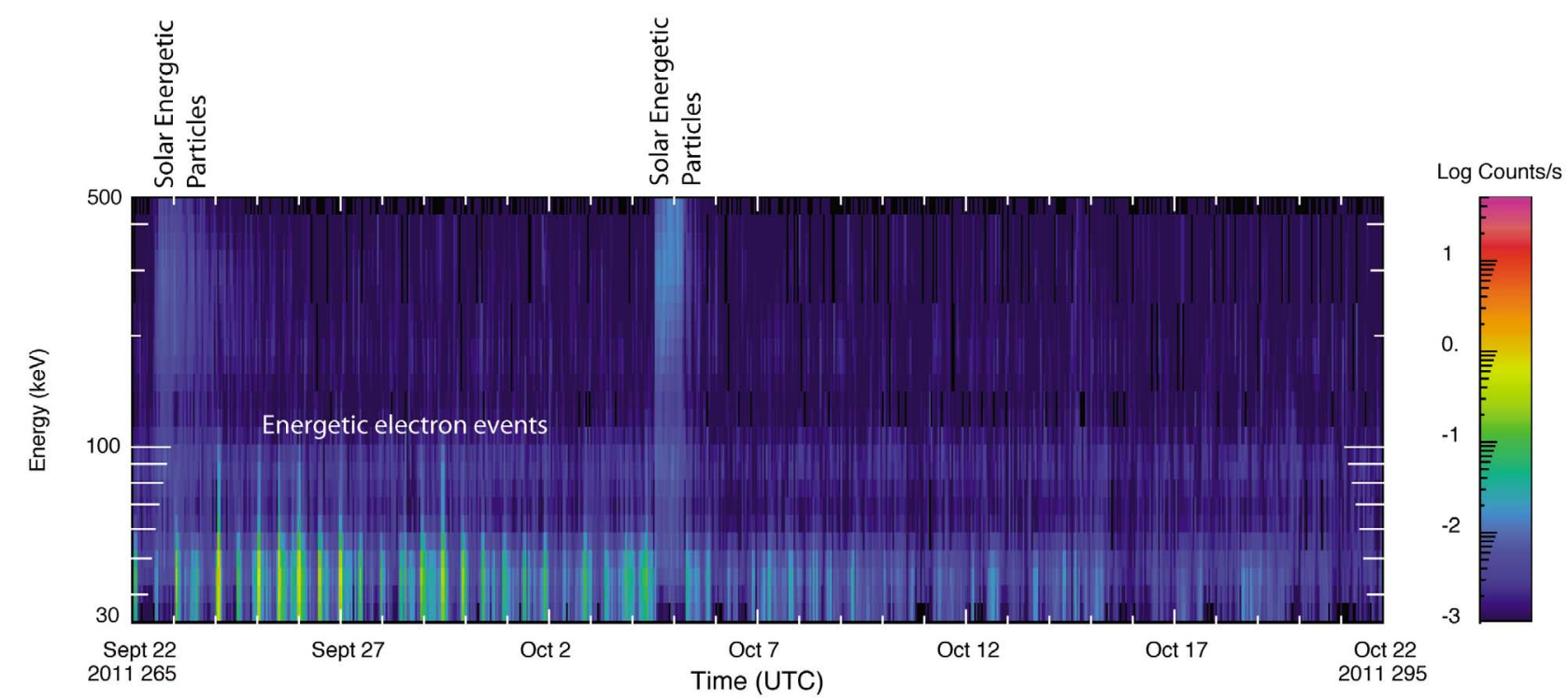

Figure 1. Energy spectrogram for the electron events observed by EPS from 22 September 2011 to 22 October 2011. MESSENGER's orbital period at Mercury during this interval was $\sim 12 \mathrm{~h}$. The electron events appear as intensity increases in the energy range 30 to $60 \mathrm{keV}$ (in orange and light blue) and generally fade into the instrument background above $100 \mathrm{keV}$. Electron event intensities, even at the lower energies, gradually dropped close to the instrument background level by 16 October, when the spacecraft orbit was in a dawn-dusk configuration.

duration (a few seconds), came in groups that spanned a few minutes to a few hours, and tended to be centered around MESSENGER's closest approach to the planet. The energy of these electrons sometimes exceeded $200 \mathrm{keV}$, but often the energy distribution exhibited a cutoff near $E=100 \mathrm{keV}$. Several of the events that were reported by Ho et al. [2011b] were observed near the magnetic equator, but most were concentrated at high northern latitudes, near spacecraft periapsis. No evidence of stably trapped high-energy particle populations similar to the Van Allen radiation belts at Earth were found. In addition, EPS found no evidence of highenergy $(>35 \mathrm{keV})$ protons above instrumental background anywhere in Mercury's environment, again in contrast to the original report from the Mariner 10 measurements [Simpson et al., 1974].

[4] Now that MESSENGER has completed one Earth year of science operations at Mercury, it is clear that these energetic $(<100 \mathrm{keV})$ electrons are common in Mercury's magnetosphere. As was reported earlier [Ho et al., 2011b], these electron events appear mainly around local midnight near the magnetic equator and at high-latitudes close to the magnetospheric cusp region on open as well as closed magnetic field lines. In this paper, we present the statistical distribution and other properties of one year's worth of these electron events, including locations and energy spectra as measured by EPS.

\section{Overview of Energetic Electron Bursts at Mercury}

[5] During its first year of orbital observations, MESSENGER was in a highly eccentric 12-h orbit. Once science operations began in March 2011, EPS and other instruments on MESSENGER recorded bursts of energetic electrons routinely on most orbits. Unlike the initial observations of energetic electron bursts reported by Ho et al. [2011b], however, the longer temporal baseline now available includes periods when such bursts are rare to absent. Figure 1 shows the EPS energy-time spectrogram for one month in the fall of 2011; the results are characteristic of most other periods to date as well. The data are hourly averaged, and the electron bursts are evident in each of the 12-h orbit periods. As illustrated in the figure, the strong electron burst activity seen during each orbit from 22 September to early October waned after 16 October. Also evident in the figure are two solar energetic particle events (on 22 September and 4 October) characterized by high energies $(>100 \mathrm{keV})$ and durations measured in days.

[6] MESSENGER's orbit around Mercury is fixed in inertial space. So, as Mercury orbits around the Sun, MESSENGER traverses through all Mercury longitudes and all magnetic local times. During one Mercury year (88 Earth days) the MESSENGER orbit alternately goes from dawndusk to noon-midnight and then repeats both configurations. In one Mercury solar day (176 Earth days) MESSENGER covers all magnetic local times twice. Because of the inclination of MESSENGER's line of apsides with periapsis at $\sim 60^{\circ}-70^{\circ} \mathrm{N}$ latitude, the two observations at a given magnetic local time occur at different distances from the planet. In the 12 months of orbital observations at Mercury, with most magnetospheric observations in the northern hemisphere, there is more variability in the record of energetic electron bursts than was apparent from the earliest orbits [Ho et al., 2011b]. During the year, there have been intervals up to several weeks in length when intense bursts were observed regularly on every orbit passage, but there have also been periods up to several days in length when energetic electron events were nearly absent or at about EPS 



Figure 2. (a) Spatial distribution of energetic electron events measured by EPS close to the planet $\left(<1 R_{\mathrm{M}}\right)$ in geographic latitude and magnetic local time. Because MESSENGER's periapsis is at a high northern latitude, there is no low-altitude coverage over the southern hemisphere south of approximately $-30^{\circ}$. Two groups of events are clearly seen in the plot; one group is concentrated at local midnight $(0 \mathrm{~h})$ and spans most latitudes sampled $\left(-30^{\circ}\right.$ to $\left.90^{\circ}\right)$, and another larger group of events seen at high latitudes $\left(30^{\circ}\right.$ to $\left.90^{\circ}\right)$ is most strongly populated near noon $(12 \mathrm{~h})$ and thins out near dusk $(18 \mathrm{~h})$. (b) A similar plot of all events that are at least above the EPS background $\left(2\right.$ counts $\mathrm{cm}^{-2} \mathrm{~s}^{-1} \mathrm{sr}^{-1} \mathrm{keV}^{-1}$ at $45 \mathrm{keV}$ ). In this population most of the events are in the same midnight and noon sectors, but they extend to lower latitudes. In addition, a weaker set of events is seen near the equator and extends over most local times. This last population is least well expressed near dawn and dusk.

background level. The periods during which few or no electron events were recorded were all when the MESSENGER orbit was in a dawn-dusk configuration (i.e., when the periapsis or apoapsis was near 6 or $18 \mathrm{~h}$ local time).

[7] MESSENGER's initial orbit following insertion on 18 March 2011 had a dawn-dusk configuration. By the time that science operations began at the end of that month, and at the time that the first energetic electron event $(27$ March) was documented [Ho et al., 2011b], the spacecraft orbit was transitioning to a noon-midnight configuration with periapsis on the nightside. By late September 2011, the spacecraft was again in an orbit phase similar to that in late March, and recurrent electron events were seen (Figure 1). Within less than a month, however, the spacecraft orbit had moved to a dawn-dusk configuration and energetic particle events became sporadic and eventually disappeared or their intensity dropped near the EPS background level. This general pattern characterizes the entire 12-month ( $\sim 4$ Mercury years, $\sim 2$ Mercury solar days) data set.

\section{Statistical Analysis of Energetic Electron Bursts at Mercury}

[8] We have systematically categorized the most intense electron events detected by EPS between 26 March 2011 and 23 March 2012. We define such an event as one in which the count rate on the 36-57 keV channel in EPS was at least a factor of 10 times the average background level (i.e., $>2 \times 10^{2} \mathrm{~cm}^{-2} \mathrm{~s}^{-1} \mathrm{sr}^{-1} \mathrm{keV}^{-1}$ ) in at least one of the six EPS detectors, which point in different directions. We also required that fluxes were simultaneously above the background count rate in at least one other detector, for at least three consecutive readouts, i.e., a minimum of $9 \mathrm{~s}$ at our finest time resolution. These criteria yielded 51 energetic electron events over the 12-month period.
[9] The locations of all of these most intense electron events that were observed within one Mercury radius $\left(R_{\mathrm{M}}\right)$ of the planet are shown in magnetic local time and geographic latitude in Figure 2a. All electron events shown are north of $30^{\circ} \mathrm{S}$, because the periapsis of the MESSENGER orbit is in the northern hemisphere and there is no sampling of the magnetosphere over much of the southern hemisphere. Two groups of events can be seen in the figure. One group is concentrated at high latitudes on the night side in the northern polar region. Another group of events is near local midnight and spans latitudes from $30^{\circ} \mathrm{S}$ to $75^{\circ} \mathrm{N}$.

[10] If we include smaller electron events, defined as those that registered at least a factor of 2 above the average background in at least one out of six detectors, the resulting set is much larger, as shown in Figure $2 b$. The distribution is generally similar to that of the higher-intensity events (i.e., one group at local midnight in the northern hemisphere and another group in the polar region), with the addition of a cluster of weak events near the geographic equator at most local times. Closer examination reveals that these events are short, with durations equal to or less than the instrument integration period $(3 \mathrm{~s})$. This group of events could be related to the low-energy, low-intensity X-ray fluorescence events observed during MESSENGER's low-latitude flybys [Ho et al., 2011a] and seen near the planet from orbital XRS observations [Starr et al., 2012]. Prompted by the lowenergy electron $(1-10 \mathrm{keV})$ events seen during the flybys, Schriver et al. [2011] reported kinetic simulation results that suggested a quasi-trapped population of low-energy $(1-10 \mathrm{keV})$ ions and electrons near Mercury's equatorial region. Hereafter, we will focus on the more intense, higherenergy electron events recorded by the EPS.

[11] The locations of all 51 of the most intense energetic electron events, coded with markers indicating their intensity, are shown in Mercury solar orbital (MSO) coordinates in Figure 3. (In MSO coordinates, $+X$ is sunward, $+Z$ is 



Figure 3. The spatial distribution of the 51 most intense electron events $\left(>200\right.$ particles $\mathrm{cm}^{-2} \mathrm{~s}^{-1} \mathrm{sr}^{-1}$ $\mathrm{keV}^{-1}$ at $45 \mathrm{keV}$ ) detected by EPS during the first 12 months of MESSENGER orbital observations, plotted in two cross-sections in MSO coordinates. Each marker denotes the location of the peak intensity during an event. The marker size increases with event intensity, and the color represents the power law index fit to the energy spectrum. Modeled locations of the magnetopause and bow shock [Slavin et al., 2009b; R. M. Winslow et al., Mercury's magnetopause and bow shock from MESSENGER observations, submitted to Journal of Geophysical Research, 2012] are shown in red and blue, respectively; the planetary outline is shown in black.

northward, and $+Y$ completes the right-handed system) The largest events were all in the vicinity of the northern cusp, near Mercury's north polar region. There were also several moderate-size events near local midnight. There were 10 events on the dayside far upstream of Mercury's bow shock.

[12] We have characterized the energy $\operatorname{spectrum} j(E)$ of each of the 51 high-intensity electron burst events by a simple power law distribution $\left(d j / d E=k E^{-\gamma}\right.$, where $\gamma$ is the power law index and $k$ is a constant) at $E<100 \mathrm{keV}$. For each event, we fit a power law spectrum to the event-averaged flux over the energy range 36 to $\sim 100 \mathrm{keV}$, since the slope is generally steeper at higher energies. (For some cases, e.g., Figure 4, the energy spectrum may be better fit by a kappa distribution than a power law.) The events are distinguished by power law index in Figure 3 . The 51 events in this study have a mean power law index of $2.51 \pm 0.85$. The 10 events that were upstream of Mercury's bow shock had a slightly softer spectral index $(2.91 \pm 0.87)$ than the remaining 41 events $(2.44 \pm 0.87)$. The spectral index at energies $>100 \mathrm{keV}$ (not shown in Figure 3 ) is generally larger, up to and greater than 3 .

\section{Examples of Energetic Electron Bursts at Mercury}

[13] Records of two of the high-latitude energetic electron bursts on 22 December 2011 are shown in Figure 4. The first event was detected just before 02:55 UTC and lasted for approximately one minute, and a second burst started at 03:01 UTC and lasted for three minutes. Multiple events were often seen during a single orbit, as shown in this instance. Another example of such behavior was the set of multiple events on 29 March 2011, shown by Ho et al. [2011b], detected both at a moderate latitude on the nightside and in the polar region during a single orbit. Both events on 22 December are characterized by short, intense bursts during which particle fluxes increased by two orders of magnitude, often within a time less than the 3-s instrument integration time. In addition, the peak fluxes during these events commonly displayed pitch angles near $90^{\circ}$ [Ho et al., 2011 b], i.e., perpendicular to the magnetic field line.

[14] As noted above, energetic electron events were also detected upstream (by as much as $1 R_{\mathrm{M}}$ ) of Mercury's dayside bow shock. From the bow shock crossings identified from observations with the MESSENGER Magnetometer, we documented 10 of these events. The largest such dayside event (24 November 2011) measured in the 12-month data set is shown in Figure 5. The outbound crossing of the Mercury bow shock was at 10:41 UTC, and the electron event started at 11:14 UTC (Figure 5), when the spacecraft was $\sim 0.8 R_{\mathrm{M}}$ from the bow shock. The pitch angle distribution for this event (Figure 6) shows that particles were flowing in a sunward direction opposite $\left(180^{\circ}\right)$ to that of the local magnetic field B. Unfortunately, EPS had good pitch angle coverage for only two of the 10 upstream events. The second was on 25 November 2011, and once again maximum intensities were nearly anti-parallel $\left(180^{\circ}\right)$ to the local magnetic field direction, i.e., particle flow away from the bow shock.

\section{Discussion}

[15] With the MESSENGER flybys and initial orbital data, Ho et al. [2011a, 2011b] established that there are indeed high fluxes of energetic electrons with energies $<100 \mathrm{keV}$ within Mercury's magnetosphere but no high-energy ions, as originally reported from Mariner 10 observations [Simpson et al., 1974]. Now that MESSENGER has collected one year's worth of orbital data, we see that electron bursts are a common occurrence within Mercury's magnetosphere. For the 51 most intense events observed during that period, most 


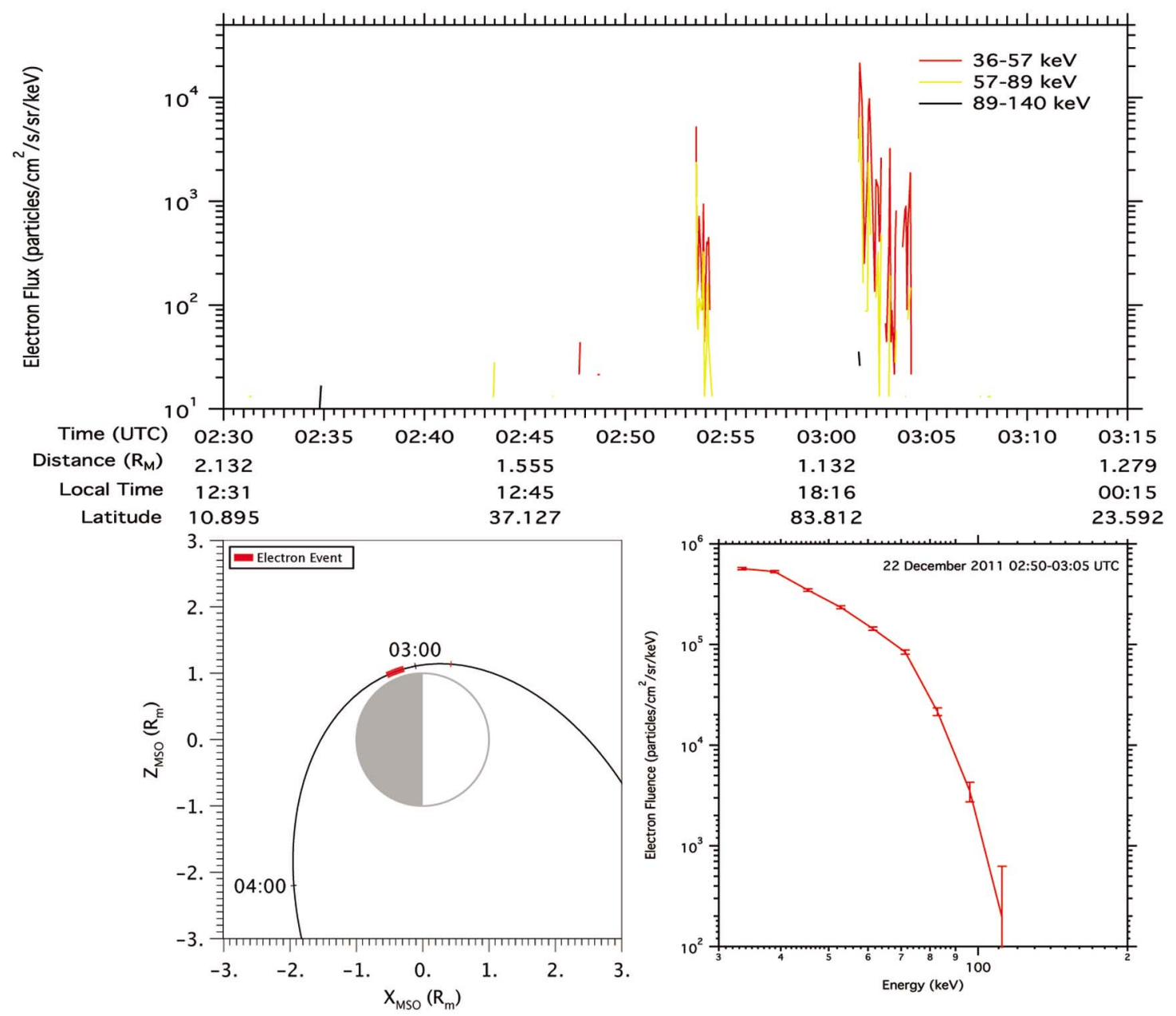

Figure 4. The intensity versus time, event-averaged energy spectrum, and location for a pair of two closely spaced high-latitude energetic electron bursts on 22 December 2011.

occurred either on the nightside at a range of latitudes or near the polar region on both the dayside and nightside. The occurrence of these electron events is also a function of magnetic local time (Figure 2). All of the largest events were detected in the noon and midnight sectors. Even among the smaller events, few were detected in either the dawn or dusk sectors. There were some small observational biases in accumulating these data. During the first of the eight noonmidnight orbital orientations over the 12 months of observations, the EPS instrument was turned off for a portion of the noon-midnight orbit due to spacecraft thermal and power constraints. Following this period, however, it was determined that there were sufficient thermal and power margins and the EPS instrument was kept powered on for the rest of the noon-midnight segments. There is also a slight bias toward observing events in the noon-midnight sector because MESSENGER spends more time within Mercury's magnetosphere in this type of orbit than when the orbit is in a dawndusk configuration. Nonetheless, because most of the events are detected close to the planet, the effect of this bias is thought to be minor.

[16] Both groups of events have similar profiles of intensity versus time. They are highly irregular and exhibit increases by up to three orders of magnitude above background within a few seconds. Events from both groups are often detected on a single orbit. The rapid rise and lack of observed velocity dispersion within the $3 \mathrm{~s}$ accumulation time prevents us from determining the source of the acceleration mechanism for these electrons. The pitch angle distributions show that the electrons were traveling more or less perpendicular to the magnetic field line. But the fact that these events were seen on a recurrent basis suggests that there is little or no strong dependence on external forcing, such as solar wind velocity or interplanetary magnetic field (IMF) direction. D. N. Baker et al. (Solar wind forcing at Mercury: WSA-ENLIL model results, submitted to Journal of Geophysical Research, 2012) compared the occurrence of electron events $(\geq 20 \mathrm{keV})$ as measured by the Neutron Spectrometer on MESSENGER with the modeled solar wind temperature and found that even although there is a weak correlation (correlation coefficient $r=0.872$ ) between the two quantities, there is considerable scatter in the data. They concluded that there may be other internal magnetospheric processes that govern the correlation.

[17] Because Mercury lacks a substantial atmosphere, these energetic electrons can follow magnetic field lines to impact the surface. The XRS instrument on MESSENGER has measured X-ray fluorescence generated by such electron 


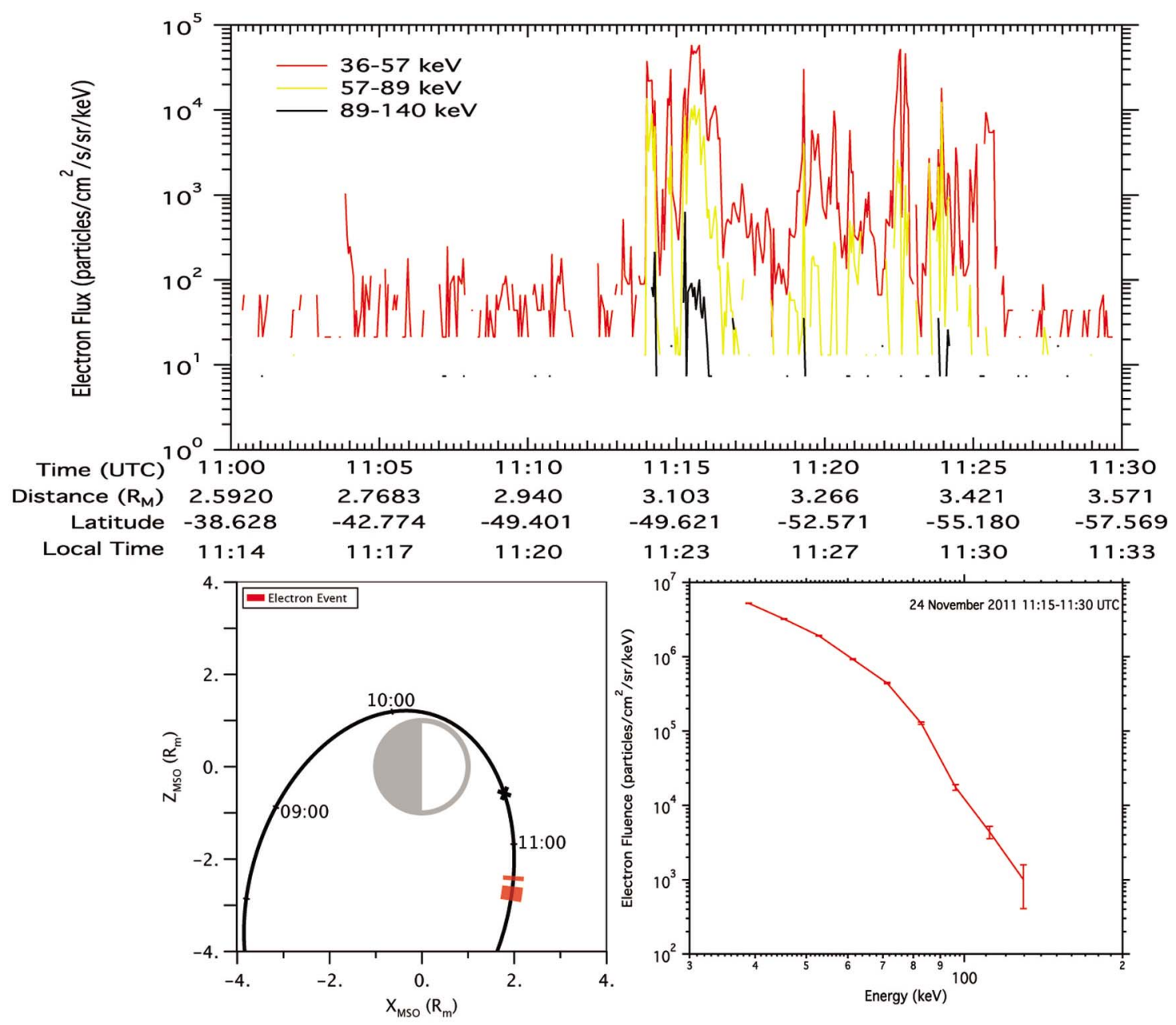

Figure 5. The largest dayside event seen outside Mercury's bow shock on 24 November 2011. The location of the Mercury bow shock crossing is marked by $\mathrm{x}$ in the lower left panel. A fit of the eventaveraged spectrum with a power law distribution over the energy range 36-100 keV gives a power law index of 3.26 .

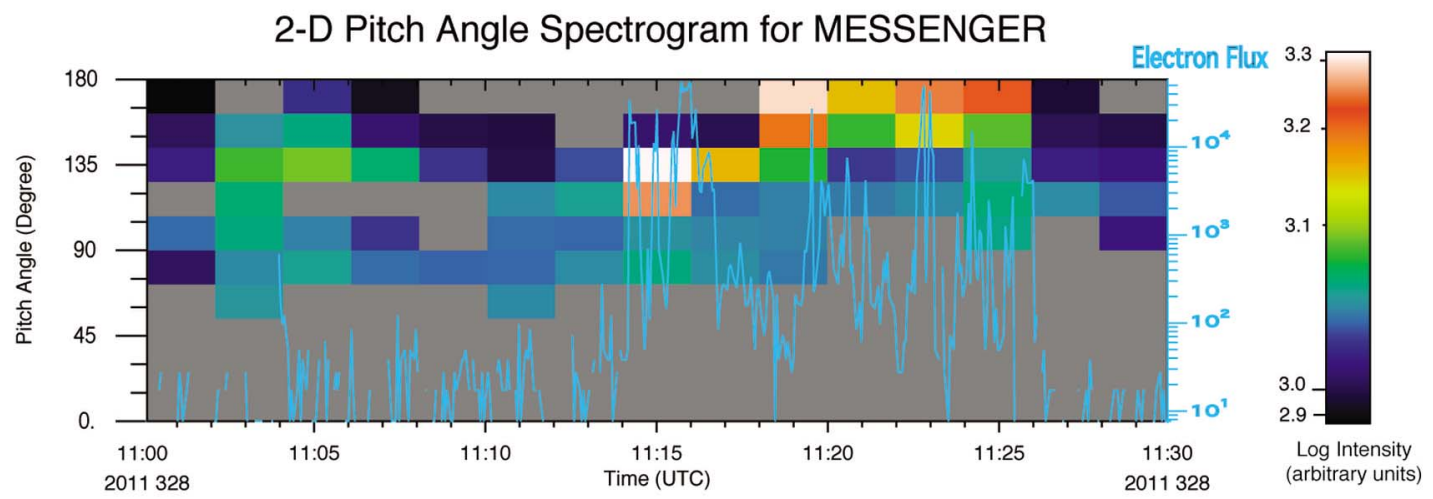

Figure 6. Pitch angle distribution and electron intensity in the 36 to $57 \mathrm{keV}$ energy channel (cyan) for the electron burst on 24 November 2011 (Figure 5). Unlike the pitch angle distributions for high-latitude nightside events, after the onset of this event at $~ 11: 14$ UTC, particles were traveling predominantly along the magnetic field line away from the bow shock until $\sim 11: 26$ UTC. 


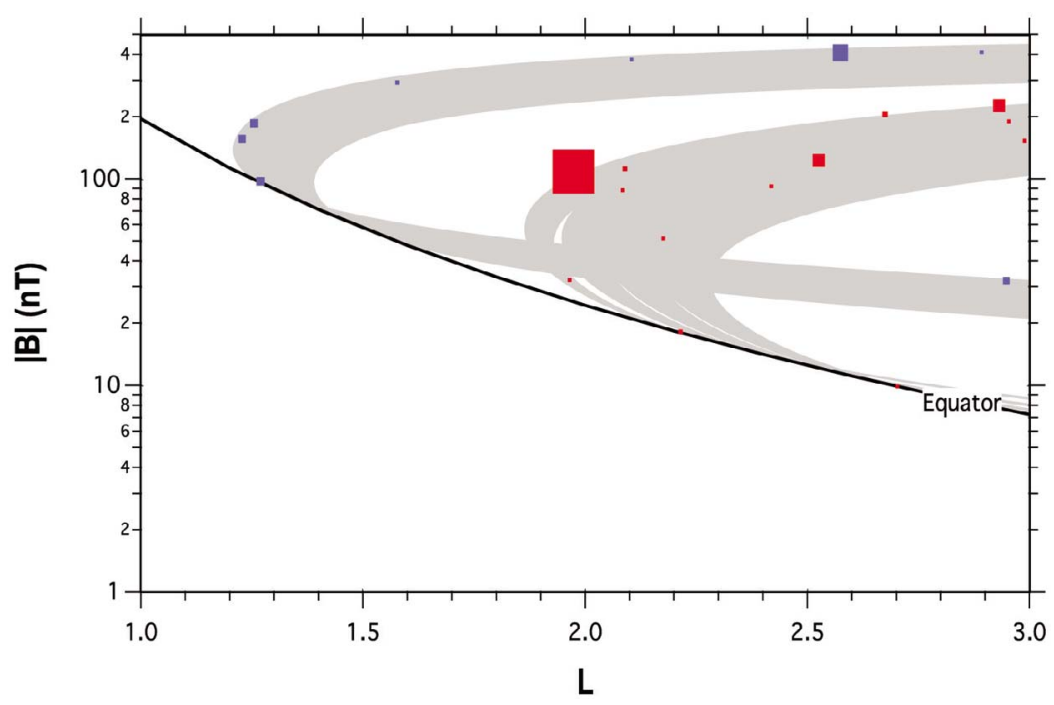

Figure 7. Locations of the electron events in $|\mathbf{B}|, L$ coordinates (see text). Shaded areas represent regions sampled by MESSENGER during the first 12 months in orbit. The red squares denote dayside events, and the blue squares denote nightside events. Symbol size increases with event intensity. The number of events in this figure is limited because many of the events are on open field lines that cannot be represented in $|\mathbf{B}|$, $L$ coordinates.

impact events [Starr et al., 2012]. From electron energy distributions measured by EPS, Starr et al. [2012] showed that surface elemental abundances inferred from such electron-induced fluorescence match abundances determined by solar-X-ray-induced fluorescence. Because of their recurrent nature, the energetic electron events observed by MESSENGER likely are an important contributor to sputtering as a source of Mercury's neutral and ionized exosphere.

[18] The coordinate system that best organizes trapped particle data in a magnetosphere is the $|\mathbf{B}|, L$ system of McIlwain [1961]. In this system, the quantity $L$ defines an axisymmetric surface of those lines of magnetic force from the dipole component of Mercury's internal field that intersect the magnetic equator at a distance $L\left(R_{\mathrm{M}}\right)$ from the dipole center. Figure 7 shows these electron events in a $|\mathbf{B}|$, $L$ system that utilizes the offset dipole model of Anderson et al. [2011] for Mercury's internal field [Ho et al., 2011b]. Most events in this analysis occurred at high latitudes where the magnetic field direction differs substantially from that of a dipole, and many field lines are likely to be open. Hence, only a few events appear on the figure. Nonetheless, the figure illustrates that there are moderate-size equatorial events on the nightside, and a large fraction of dayside events are far from the equatorial region, but most likely outside the dayside magnetopause.

[19] Because of the similarity of the most intense electron bursts seen at high latitudes and near local midnight, we infer that the electrons in both types of events were accelerated by the same mechanism. Moreover, the recurrent and burst-like nature of these events indicates that the acceleration mechanism is efficient, operates on timescales of seconds, and is most likely located within Mercury's magnetosphere not far from the planet. The observed latitudinal variations in burst locations could indicate that the source position varies with time, the source occupies a broad region, or the instantaneous field configuration that maps field lines to the acceleration region varies with time.

[20] At Earth, magnetospheric substorms are a powerful mechanism for particle acceleration that is able to accelerate plasma to energies up to hundreds of $\mathrm{keV}$ [Baker et al., 1996]. During a substorm at Earth, magnetic energy is unloaded through reconnection in the tail lobes, resulting in the ejection of plasmoids; high-speed sunward and antisunward jetting of hot plasma; acceleration and injection of charged particles, both electrons and protons, into the inner magnetosphere; and field-aligned currents flowing between the tail and the high-latitude atmosphere where aurorae are produced [Angelopoulos et al., 2008]. Although substorm activity has been observed at Mercury [e.g., Sundberg et al., 2012], we have not been able to establish to date a connection between such magnetic activity and electron acceleration.

[21] The measurements presented here require a lower energy release than is typical for a reconnection event in the Earth's magnetosphere. During MESSENGER's second Mercury flyby, when the IMF was southward (a preferred IMF orientation for reconnection at Mercury, as at Earth), the inferred cross-magnetosphere electric potential was about $30 \mathrm{kV}$ [Slavin et al., 2010], but the variation in transfer of magnetic flux to the tail was by more than a factor of $\sim 3$, from 3 to $\sim 9.5 \mathrm{MWb}$, suggesting that cross-tail fields can vary by at least the same factor [Sundberg et al., 2012]. Thus, energies in excess of about $100 \mathrm{keV}$ would not be uncommon, consistent with the spectra that were observed for most of the electron bursts. However, these events recur on a regular basis, so if substorm-associated reconnection is the acceleration mechanism, substantial reconnection within the Mercury magnetosphere must be occurring most of the time.

[22] At Earth, plasma sheet particles are accelerated to energies above the electrostatic potential across the magnetosphere [Krimigis and Sarris, 1979]. A possible model 
invoking tearing-mode instability with a small magnetic field component normal to the current sheet can be shown to produce explosive reconnection that is short-lived (seconds) and dissipates the stored magnetic energy rapidly [Galeev et al., 1978; Baker et al., 1984, 1996]. There have been instances at Earth, however, where strong electric field pulses were seen at high latitudes together with impulsive events in the plasma sheet [Krimigis and Sarris, 1979]. Such occurrences could well be operating within Mercury's magnetosphere and could give rise to the observed electron bursts. Finally, there is the possibility that direct impingement of solar wind plasma on the planet's surface through the cusps may generate potentials of tens of $\mathrm{keV}$ that could accelerate the electrons observed near polar cusp field lines.

[23] The events observed upstream of Mercury's bow shock have similarities to a class of events observed at Earth. Their average spectral index $(\gamma \sim 2.9)$, and the electron flow along the magnetic field away from the bow shock, in the two events for which we were able to observe pitch angle distribution, appear to be similar to events that are commonly observed in a similar setting upstream of Earth's bow shock [Lin et al., 1974] during which electrons are accelerated via shock-drift [Armstrong et al., 1985] at the perpendicular bow shock and travel upstream into the solar wind. We examined in detail the upstream IMF conditions for all 10 of the upstream electron events, and we found that five of those events (including that on 25 November 2011) are associated with regions that have a quasi-parallel bow shock geometry, four with quasi-perpendicular geometry (including the event shown in Figure 5), and one with a highly fluctuating shock crossing. At Earth, electrons (and ions) have been observed streaming away from the bow shock in regions that generally have a quasi-perpendicular geometry [Anderson et al., 1979; Kasaba et al., 2000]. It appears that, at times, processes analogous to those at Earth's foreshock region can be found at Mercury's bow shock. For nominal values of solar wind speed $(400 \mathrm{~km} / \mathrm{s})$, characteristic drift distance along Mercury's bow shock $\left(2 R_{M}\right)$, and IMF field strength $(50 \mathrm{nT})$, we estimate that the energy gain by shockdrift at Mercury's bow shock [Decker, 1988] is $\sim 100 \mathrm{keV}$, which is consistent with the energies of the electrons observed by MESSENGER.

\section{Conclusions}

[24] MESSENGER has, as of this writing, completed 12 months of orbital observations at Mercury. The Energetic Particle Spectrometer has continued to observe energetic electron bursts on nearly all of its 12-h orbits within Mercury's magnetosphere. Most of these events are observed around local midnight and at high latitudes, and such events are rare to absent in the dawn and dusk sectors. A smaller, weaker set of events that are barely above instrumental background was also seen in the equatorial region at most local times. This weaker set of events may be the high-energy tail of the $1-10 \mathrm{keV}$ quasi-trapped population of electrons in Mercury's equatorial regions that were seen in the hybrid simulation of Schriver et al. [2011]. Both the high-latitude and nightside events are similar in pitch angle, intensity, and spectral index. They are most likely being accelerated by the same acceleration mechanism, and variations in the latitude at which the events were observed could be due to temporal variations in either the source location or the instantaneous magnetic field configuration that maps field lines to the source. If the acceleration mechanism is similar to reconnection, one of the proposed acceleration mechanisms that accompanies terrestrial substorms, the implied reconnection rate is quite high, since we observe burst events on most orbits and there are often several bursts on a given orbit. A high rate of reconnection at Mercury is consistent with the findings of Slavin et al. [2009a, 2010] from magnetic field observations. The group of energetic electron events seen upstream of Mercury's bow shock has pitch angle distributions that are different from those of the high-latitude and nightside events. The pitch angle distributions and energy considerations for nominal IMF and solar wind conditions are consistent with shock-drift acceleration at Mercury's bow shock and escape of the particles upstream from Mercury.

[25] Acknowledgments. We thank the MESSENGER team for the development, cruise, orbit insertion, and Mercury orbital operations of the MESSENGER spacecraft. The MESSENGER mission is supported by the NASA Discovery Program under contracts NAS5-97271 to the Johns Hopkins University Applied Physics Laboratory and NASW-00002 to the Carnegie Institution of Washington.

[26] Masaki Fujimoto thanks the reviewers for their assistance in evaluating this paper.

\section{References}

Anderson, B. J., C. L. Johnson, H. Korth, M. E. Purucker, R. M. Winslow, J. A. Slavin, S. C. Solomon, R. L. McNutt Jr., J. M. Raines, and T. H. Zurbuchen (2011), The global magnetic field of Mercury from MESSENGER orbital observations, Science, 333, 1859-1862, doi:10.1126/ science. 1211001

Anderson, K. A., R. P. Lin, F. Martel, C. S. Lin, G. K. Parks, and H. Rème (1979), Thin sheets of energetic electrons upstream from the Earth's bow shock, Geophys. Res. Lett., 6, 401-404, doi:10.1029/GL006i005p00401.

Andrews, G. B., et al. (2007), The Energetic Particle and Plasma Spectrometer instrument on the MESSENGER spacecraft, Space Sci. Rev., 131, 523-556.

Angelopoulos, V., et al. (2008), Tail reconnection triggering substorm onset, Science, 321, 931-935, doi:10.1126/science/1160495.

Armstrong, T. P., S. M. Krimigis, and L. J. Lanzerotti (1975), A reinterpretation of the reported energetic particle fluxes in the vicinity of Mercury, J. Geophys. Res., 80, 4015-4017.

Armstrong, T. P., L. J. Lanzerotti, and S. M. Krimigis (1979), Comment on

"Electron calibration of instrumentation for low-energy high-intensity particle measurements at Mercury" by Christon, Daly, Eraker, Perkins, Simpson, and Tuzzolino, J. Geophys. Res., 84, 4468-4469.

Armstrong, T. P., M. E. Pesses, and R. B. Decker (1985), Shock drift acceleration, in Collisionless Shocks in the Heliosphere: Reviews of Current Research, edited by B. T. Tsurutani and R. G. Stone, Geophys. Monogr. Ser., vol. 35, pp. 271-285, AGU, Washington, D. C.

Baker, D. N., S.-I. Akasofu, W. Baumjohann, J. W. Bieber, D. H. Fairfield, E. Q. Hones Jr., B. Mauk, R. L. McPherron, and T. E. Moore (1984), Substorms in the magnetosphere, in Solar Terrestrial Physics: Present and Future, edited by D. M. Butler and K. Papadopoulos, NASA Ref. Publ., RP-1120, 8.1-8.55.

Baker, D. N., T. I. Pulkkinen, V. Angelopoulos, W. Baumjohann, and R. L. McPherron (1996), The neutral line model of substorms: Past results and present view, J. Geophys. Res., 101, 12,995-13,010.

Christon, S. P. (1989), Plasma and energetic electron flux variations in the Mercury $1 \mathrm{C}$ event: Evidence for a magnetospheric boundary layer, J. Geophys. Res., 94, 6481-6505.

Decker, R. B. (1988), Computer modeling of test particle acceleration at oblique shocks, Space Sci. Rev., 48, 195-262.

Galeev, A. A., F. V. Coroniti, and M. Ashour-Abdala (1978), Explosive tearing mode reconnection in the magnetospheric tail, Geophys. Res. Lett., 5, 707-710.

Ho, G. C., R. D. Starr, R. E. Gold, S. M. Krimigis, J. A. Slavin, D. N. Baker, B. J. Anderson, R. L. McNutt Jr., L. R. Nittler, and S. C. Solomon (2011a), Observations of suprathermal electrons in Mercury's magnetosphere during the three MESSENGER flybys, Planet. Space Sci., 59, 2016-2025, doi:10.1016/j.pss.2011.01.011. 
Ho, G. C., et al. (2011b), MESSENGER observations of transient bursts of energetic electrons in Mercury's magnetosphere, Science, 333 , 1866-1868, doi:10.1126/science.1211001.

Kasaba, Y., H. Matsumoto, Y. Omura, R. R. Anderson, T. Mukai, Y. Saito, T. Yamamoto, and S. Kokubun (2000), Statistical studies of plasma waves and backstreaming electrons in the terrestrial electron foreshock observed by Geotail, J. Geophys. Res., 105, 79-103, doi:10.1029/ 1999JA900408.

Krimigis, S. M., and E. T. Sarris (1979), Energetic particle bursts in the Earth's magnetotail, in Dynamics of the Magnetosphere, edited by S.-I. Akasofu, pp. 599-630, D. Reidel, Dordrecht, Netherlands.

Lin, R. P., C.-I. Meng, and K. A. Anderson (1974), 30- to 100-keV protons upstream from the Earth's bow shock, J. Geophys. Res., 79, 489-498.

McIlwain, C. E. (1961), Coordinates for mapping the distribution of magnetically trapped particles, J. Geophys. Res., 66, 3681-3691.

McNutt, R. L., Jr., S. C. Solomon, P. D. Bedini, E. J. Finnegan, D. G. Grant and the MESSENGER Team (2010), The MESSENGER mission Results from the first two Mercury flybys, Acta Astronaut., 67, 681-687.

Schriver, D., et al. (2011), Quasi-trapped ion and electron populations at Mercury, Geophys. Res. Lett., 38, L23103, doi:10.1029/2011GL049629.
Simpson, J. A., J. H. Eraker, J. E. Lamport, and P. H. Walpole (1974), Electrons and protons accelerated in Mercury's magnetic field, Science, $185,160-166$.

Slavin, J. A., et al. (2009a), MESSENGER observations of magnetic reconnection in Mercury's magnetosphere, Science, 324, 606-610, doi:10.1126/science.1172011.

Slavin, J. A., et al. (2009b), MESSENGER observations of Mercury's magnetosphere during northward IMF, Geophys. Res. Lett., 36, L02101, doi:10.1029/2008GL036158

Slavin, J. A., et al. (2010), MESSENGER observations of extreme loading and unloading of Mercury's magnetic tail, Science, 329, 665-668, doi:10.1126/science. 1159081

Solomon, S. C. (2011), A new look at the planet Mercury, Phys. Today, 64(1), 50-55.

Starr, R. D., D. Schriver, L. R. Nittler, S. Z. Weider, P. K. Byrne, G. C. Ho, E. A. Rhodes, C. E. Schlemm II, S. C. Solomon, and P. M. Trávníček (2012), MESSENGER detection of electron-induced X-ray fluorescence from Mercury's surface, J. Geophys. Res., 117, E00L02, doi:10.1029/ 2012JE004118

Sundberg, T., et al. (2012), MESSENGER observations of dipolarization events in Mercury's magnetotail, J. Geophys. Res., doi:10.1029/ 2012JA017756, in press. 